\title{
The effect of rehabilitation in patients with polyneuropathy induced by occupational intoxication with n-hexane: a report of 9 cases
}

\author{
Yun Sun $^{1 \#} \wedge$, Xiaohong Wu ${ }^{1 \#}$, Jinxiu Chen ${ }^{1 \#}$, Shuang Wei ${ }^{1}$, Fang $\mathrm{Ji}^{2}$, Rouna $\mathrm{Wu}^{1}$, Lin Mao ${ }^{1}$, Wangxiao Bao ${ }^{1 \wedge}$, \\ Yankai $\mathrm{Wen}^{3} \wedge$, Zuobing Chen ${ }^{1}$ \\ ${ }^{1}$ Department of Rehabilitation Medicine, The First Affiliated Hospital, Zhejiang University, Hangzhou, China; ${ }^{2}$ Department of Neurology, The First \\ Affiliated Hospital, Zhejiang University, Hangzhou, China; ${ }^{3}$ Department of Anesthesiology, McGovern Medical School, University of Texas Health \\ Science Center at Houston, TX, USA \\ Contributions: (I) Conception and design: Y Sun; (II) Administrative support: Z Chen; (III) Provision of study materials or patients: Y Sun, X Wu, J \\ Chen, S Wei, F Ji, R Wu, L Mao; (IV) Collection and assembly of data: Y Sun, X Wu, J Chen, S Wei; (V) Data analysis and interpretation: Y Sun, J \\ Chen, S Wei, W Bao, Y Wen; (VI) Manuscript writing: All authors; (VII) Final approval of manuscript: All authors. \\ "These authors contributed equally to this work. \\ Correspondence to: Zuobing Chen. Department of Rehabilitation Medicine, The First Affiliated Hospital, Zhejiang University, Qingchun Road NO. \\ 79, Hangzhou, China. Email: czb1971@zju.edu.cn.
}

Background Polyneuropathy caused by n-hexane in its occupational settings is diagnosed with bilaterally symmetrical sensory and motor abnormalities. However, no effective treatments are available.

Methods: We use the detailed physical, neurological examinations, rehabilitation assessment scale, and electrophysiological examinations at hospital admission and six months' follow up to assess the effect of a rehabilitation program on peripheral nerve injury caused by n-hexane nine patients.

Results: We found that all patients complained about sensory issues of numbness in the distal extremities and decreasing strength with a decreased locomotion speed and gait abnormalities on admission, which is following the result of electrophysiological examinations. After they underwent a hospitalized rehabilitation program for 6 months, all of them showed a significant improvement in muscle strength, balance, deep tendon reflex, walking speed, and Barthel index, which showed a significant improvement in their athletic ability, although some patients still had gait abnormalities. According to the electrophysiological test results, nine patients had increased motor conduction velocities and amplitudes and shortened distal latencies in the four limbs compared with the results upon admission or one month later. However, only some indexes of sensory nerve conduction showed significant differences. With the recovery of movement and sensory function, they could live entirely independently and even return to work.

Conclusions: We suggest that early general physical evaluation with electrophysiological examinations and comprehensive rehabilitation, including different modalities, therapeutic exercise, nerve mobilization, gait training, occupational therapy, traditional Chinese medicine treatment, and patient education, are essential so that patients can perform activities of daily living independently and return to work early.

Keywords: N-hexane; rehabilitation; polyneuropathy; occupational intoxication

Submitted Sep 17, 2020. Accepted for publication Nov 19, 2020.

doi: 10.21037/apm-20-2176

View this article at: http://dx.doi.org/10.21037/apm-20-2176

^ ORCID: Yun Sun: 0000-0002-8956-0789; Wangxiao Bao: 0000-0001-7290-7873; Yankai Wen: 0000-0002-8144-1515. 


\section{Introduction}

N-hexane $\left(\mathrm{C}_{6} \mathrm{H}_{14}\right)$, a volatile organic compound widely used in many industries, can serve as a detergent for printing, hardware and electronic device manufacturing, adhesive for leather and shoe or bag thinner for the paint industry, and crude oil extractor in food manufacturing. Because it is widely used in various industrial processes, $n$-hexane is also notorious for its neurotoxicity in occupational settings, with case reports of neuropathy appearing in the medical literature as early as 1964. Exposure to n-hexane may occur via the inhalation of toxic vapors or a percutaneous route. Along with industrialization, the outbreak of occupational $\mathrm{n}$-hexane poisoning in China has increased and caused a great deal of concern.

$\mathrm{N}$-hexane polyneuropathy is the main clinical feature and diagnostic indicator of this disease. Polyneuropathy is diagnosed with bilaterally symmetrical sensory abnormalities, including numbness and tingling sensations in the fingers and toes, and motor disorders of inferior motor neurons with symptoms of weakness in the distal segments of the legs and neurogenic damage related to electrophysiological changes. However, no effective treatment is available. The early recognition of $n$-hexane toxicity manifestations is essential to prevent the adverse effects of $n$-hexane from developing. Avoiding the toxin and exercise are efficient management strategies for patient rehabilitation. As neuropathy caused by n-hexane is characterized by sensory loss and weakness in the lower extremities, the treatment goals are to manage the condition causing neuropathy and to relieve the symptoms. However, few reports about the effect of rehabilitation on polyneuropathy are induced by occupational intoxication with n-hexane.

In the current study, we reported nine patients working in the same factory, and we focused on the effect of the rehabilitation program on the peripheral nerve injuries caused by n-hexane.

We present the following article in accordance with the STROBE reporting checklist (available at http://dx.doi. org/10.21037/apm-20-2176).

\section{Methods}

The study was conducted in accordance with the Declaration of Helsinki (as revised in 2013). The study was approved by Clinical Research Ethics Committee of the First Affiliated Hospital, Zhejiang University School of
Medicine (NO.: IIT20200475A) and informed consent was obtained from all the patients.

\section{Patients}

We enrolled nine patients diagnosed with polyneuropathy induced by occupational intoxication with n-hexane at the Department of Rehabilitation Medicine of First Affiliated Hospital, Zhejiang University, from February 2018 to October 2018 (The institutional review board approved the study's design: IIT20200475A). We collected the medical history of 9 patients before and during the rehabilitation, including history, medical records, medication, physical examinations, laboratory indicators, rehabilitation treatments, and other related informations.

Detailed physical, neurological examinations, and rehabilitation assessment scales were performed at hospital admission and six months of the treatment. Clinical therapy includes neural nutrients or regeneration medications, including mecobalamin, a mouse nerve growth factor, gangliosides, reduced glutathione, fursultiamine, vitamin C, vitamin B1, and anti-inflammatory therapy with hexadecadrol.

\section{Nerve electrophysiological examination}

Electrophysiological examinations were checked at admission and after 1, 3, and 6 months of treatment. The tests included the following: (I) motion conduction velocity (MCV), compound muscle action potential amplitude (CMAP), and distal latency (DAL) of the median, ulnar, peroneal, and tibial nerves; (II) sensory conduction velocity (SCV), sensory nerve action potential amplitude (SNAP), DAL of the median, ulnar, superficial peroneal and sural nerves. A measurement method and the normal value criterion follow the diagnosis of occupational chronic n-hexane poisoning (GBZ 84-2017) (1).

\section{Statistical analysis}

The statistical package SPSS16.0 was used to perform the statistical analysis among groups. Unless otherwise noted, the results were expressed as mean \pm standard deviation. Parametric data were analyzed using a $t$-test, analysis of variance, and other statistical processing. A probability value of $<0.05$ and $<0.01$ was used as the critical level of significance for all statistical tests. 


\section{Results}

Nine patients, including six females and three males, with ages ranging from 28 to 50 years old, worked in the same leather processing company, and did not have a history of alcohol or drug consumption. Seven of the patients' processed garments brushed glue and sewed, and handled 30-40 pieces of clothing daily. One patient (patient No. 1) examined and modified the completed garments, and he or she processed 500-600 clothes daily. The remaining patient (No. 2) was the workshop manager, and he or she brushed glue, sewed, and stacked clothes for a long time. All patients worked for 9-12 or more hours daily for 2-8 months. During working hours, the office space was closed for a long time without ventilation.

From late December 2017 to late January 2018, 9 patients showed different degrees of tiredness, symptoms, susceptibility to developing the common cold, and weakness in both legs. There was a trend in symptom development from mild to severe symptoms, including slow walking, numbness in both hands and legs, the inability to hold a bowl or wring a towel, swelling in the legs, and difficulty climbing stairs. Some patients experienced nausea, vomiting, dizziness, and constipation. The patients did not have a fever, limb pain, chest tightness, or shortness of breath. No patients had a history of allergies or contact with other toxicants.

After the onset of the illness, nine patients went to the local hospital for treatment. However, the diagnosis was unclear, and they were not treated and were referred to a superior hospital. Some patients were suspected of having acute inflammatory demyelinating polyneuropathy (patient No. 1) or Guillain-Barre syndrome (patient No. 2) and were administered intravenous immunoglobulin and cortisone treatment. After over three hospital visits, one patient underwent an electrophysiological examination until nearly two months later, and a relationship between the disease and a history of n-hexane contact was found. The diagnosis of peripheral polyneuropathy induced by n-hexane poisoning was finally confirmed. Nine patients came to the emergency department or neurology department for clinical therapy during March 2018. After 12 to 23 days, nine patients were transferred to the rehabilitation department for an average of 225 days of rehabilitation therapy.

The examinations involve identifying and defining the patient's impairment(s) and determining appropriate intervention programs for them. The examinations comprised three components: the patient history, systems review, and tests and measurements. The examinations were first conducted after patient referral or initial entry (direct access) and continued throughout care. Frequent reexaminations allow the therapists at our hospital to test progress and modify interventions as needed. According to the diagnosis of occupational chronic n-hexane poisoning (GBZ 84-2017) (1), 9 patients were divided into severe degree of $\mathrm{n}$-hexane poisoning, which is based on the clinical manifestations, sensation, muscle strength, Achilles tendon reflex and electromyography results.

After these physical examinations were conducted, we found that all patients complained about sensory issues, six experienced numbness in the distal extremities. Decreasing strength was clear in the motor function test in all patients, especially the distal segments. For most, a decreased locomotion speed and gait abnormalities were clear. For these patients, we used the modified Barthel Index to assess their level of functional performance. Only two patients showed significantly reduced activity performance (Barthel index scores of 25 and 30), while others showed less severe effects because of $n$-hexane.

During therapy, some complications developed: seven patients developed a pulmonary infection, five patients developed cough and sputum, two patients developed constipation, one patient developed venous thrombosis of the lower extremity, four patients developed hypokalemia, and six patients developed mild liver function injury.

After they underwent a hospitalized rehabilitation program, all patients showed significant improvements. All details are shown below in Table 1. After six months of treatment, all patients' muscle strength returned to a normal level, but the strength of the distal muscle groups in the lower extremities of the two patients with relatively severe conditions did not return to normal because of the severity of the injuries of the patients. Seven of these patients had varying degrees of sensory impairment, and a normal level of sensation was restored in only one of them; the EMG results showed changes in the conduction velocity and amplitude of the signals of the sensory branches of the peripheral nerves. In the balance function assessment, these patients showed a near-normal status after treatment. Although some patients still had gait abnormalities, after six months' rehabilitation, all of them showed a significant improvement in muscle strength, balance, deep tendon reflex, walking speed, and Barthel index $(\mathrm{P}<0.01$, details in Table 1), which showed a significant improvement in their athletic ability. With the recovery of movement and sensory 
Table 1 Rehabilitation evaluation of 9 patients

\begin{tabular}{|c|c|c|c|}
\hline Peripheral nerve & Pre $(n=9)$ & Post $(n=9)$ & $P$ value \\
\hline \multicolumn{4}{|l|}{ Muscle strength } \\
\hline Shoulder flexion & $3.11 \pm 0.60$ & $4.89 \pm 0.33$ & 0.0039 * \\
\hline Elbow flexion & $3.22 \pm 0.67$ & $5.00 \pm 0.00$ & $0.0039^{*}$ \\
\hline Wrist dorsiflexion & $3.00 \pm 0.50$ & $5.00 \pm 0.00$ & $0.0039^{*}$ \\
\hline Fingers flexion & $2.56 \pm 0.88$ & $4.89 \pm 0.33$ & $0.0039^{*}$ \\
\hline Hip flexion & $2.89 \pm 0.78$ & $5.00 \pm 0.00$ & $0.0039^{\star}$ \\
\hline Hip extension & $2.56 \pm 0.73$ & $5.00 \pm 0.00$ & $0.0039^{*}$ \\
\hline Knee extension & $2.78 \pm 0.67$ & $5.00 \pm 0.00$ & $0.0039^{*}$ \\
\hline Ankle & $1.78 \pm 0.67$ & $4.89 \pm 0.33$ & $0.0039^{*}$ \\
\hline Ankle dorsiflexion & $1.56 \pm 0.53$ & $4.78 \pm 0.44$ & $0.0039^{*}$ \\
\hline Toes flexion & $1.33 \pm 0.87$ & $4.66 \pm 0.71$ & $0.0039^{*}$ \\
\hline Toes extension & $1.33 \pm 0.87$ & $4.66 \pm 0.71$ & $0.0039^{\star}$ \\
\hline \multicolumn{4}{|l|}{ Balance } \\
\hline Sitting & $2.11 \pm 1.05$ & $3.00 \pm 0.00$ & 0.0625 \\
\hline Standing & $1.33 \pm 0.87$ & $2.89 \pm 0.33$ & $0.0039^{\star}$ \\
\hline \multicolumn{4}{|l|}{ Deep tendon reflex } \\
\hline Patellar tendon & $0.22 \pm 0.44$ & $1.78 \pm 0.44$ & $0.0039^{\star}$ \\
\hline Achilles tendon & $0.22 \pm 0.44$ & $1.78 \pm 0.44$ & $0.0039^{\star}$ \\
\hline Biceps & $1.00 \pm 1.00$ & $1.89 \pm 0.33$ & 0.0625 \\
\hline Triceps & $1.11 \pm 1.05$ & $1.89 \pm 0.33$ & 0.1250 \\
\hline Walking speed (cm/s) & $0.46 \pm 0.27$ & $1.80 \pm 0.25$ & $0.0039^{\star}$ \\
\hline Barthel Index & $67.78 \pm 28.19$ & $99.44 \pm 1.67$ & $0.0039^{*}$ \\
\hline
\end{tabular}

Data were shown as mean \pm standard deviation. $P$ values were calculated by Wilcoxon matched-pairs signed-rank test. ${ }^{*} \mathrm{P}<0.01$ compared with "pre" group, which is collected on admission.

function, they could live entirely independently and even return home and return to work.

All patients underwent electrophysiological examinations to confirm their diagnoses and 1-, 3- and 6-month followups (Table 2). According to the electrophysiological test results, nine patients (100\%) have sensorimotor neuropathy with demyelination damage. All patients' motor and sensory conduction studies revealed that the upper and lower extremities were nearly affected equally. Nerve conduction studies show decreased motor conduction velocities and amplitudes and the prolongation of distal latencies upon admission. During the follow-up period, the motor nerves' electrophysiological results one month later in the nine patients were worse than the results upon admission, but some patients showed improvements three months later. All patients had progressive symptoms six months later, with increased motor conduction velocities and amplitudes and shortened distal latencies in the four limbs compared with the results upon admission or one month later. However, only some indexes of sensory nerve conduction show significant differences.

\section{Discussion}

The polyneuropathy induced by n-hexane poisoning is a chronic paroxysm disease. Nine patients described in this paper had been working in a poorly ventilated, small workshop with inadequate hygienic conditions and had a history of chronic exposure to the glue that contained n-hexane for 9-12 hours per day 2-8 months. Patient No. 1 handled 500-600 completed garments, 15 to 20 times the number of garments that other colleagues handled. Although patient No. 2 handled various garments, he stayed in a place where the clothes were stacked for the entire workday. Patients No. 1 and No. 2 showed more serious motorsensory dysfunction with significantly reduced activity performance (Barthel index scores of 25 and 30) than other 7 cases. They were considered having experienced the highest level of overexposure, which concludes that a high concentration of n-hexane with poor ventilation had a direct relationship with the severity of the patient's condition. The severity of neuropathy is related to the intensity, duration, and frequency of $\mathrm{n}$-hexane exposure. Poor ventilation in the workplace has been one of the significant factors in all cases reported worldwide, and a similar situation is found in our study.

$\mathrm{N}$-hexane poisoning is exceedingly difficult to diagnose. Nine patients did not seek medical care until two months after the onset of the weakness. First, the early stage symptoms are hard to recognize, as they can include fatigue, susceptibility to the common cold, cough and sputum, nausea and vomiting, dizziness, or constipation only. The first distinguishing symptom of the disease is paraesthesia. Sensorial and motor deficits develop within days or weeks. In our study, when the nine patients visited a doctor, the primary clinical findings were slowly progressing, and symmetrical muscle weakness was observed in the distal extremities, but there was no evidence of CNS involvement. Second, in the absence of a history of n-hexane exposure, it is difficult for doctors to diagnose at the first visit, with some cases being misdiagnosed as acute inflammatory demyelinating polyneuropathy or Guillain-Barre syndrome. 
Table 2 Electrophysiological findings for nine patients on admission to 6-month follow-up

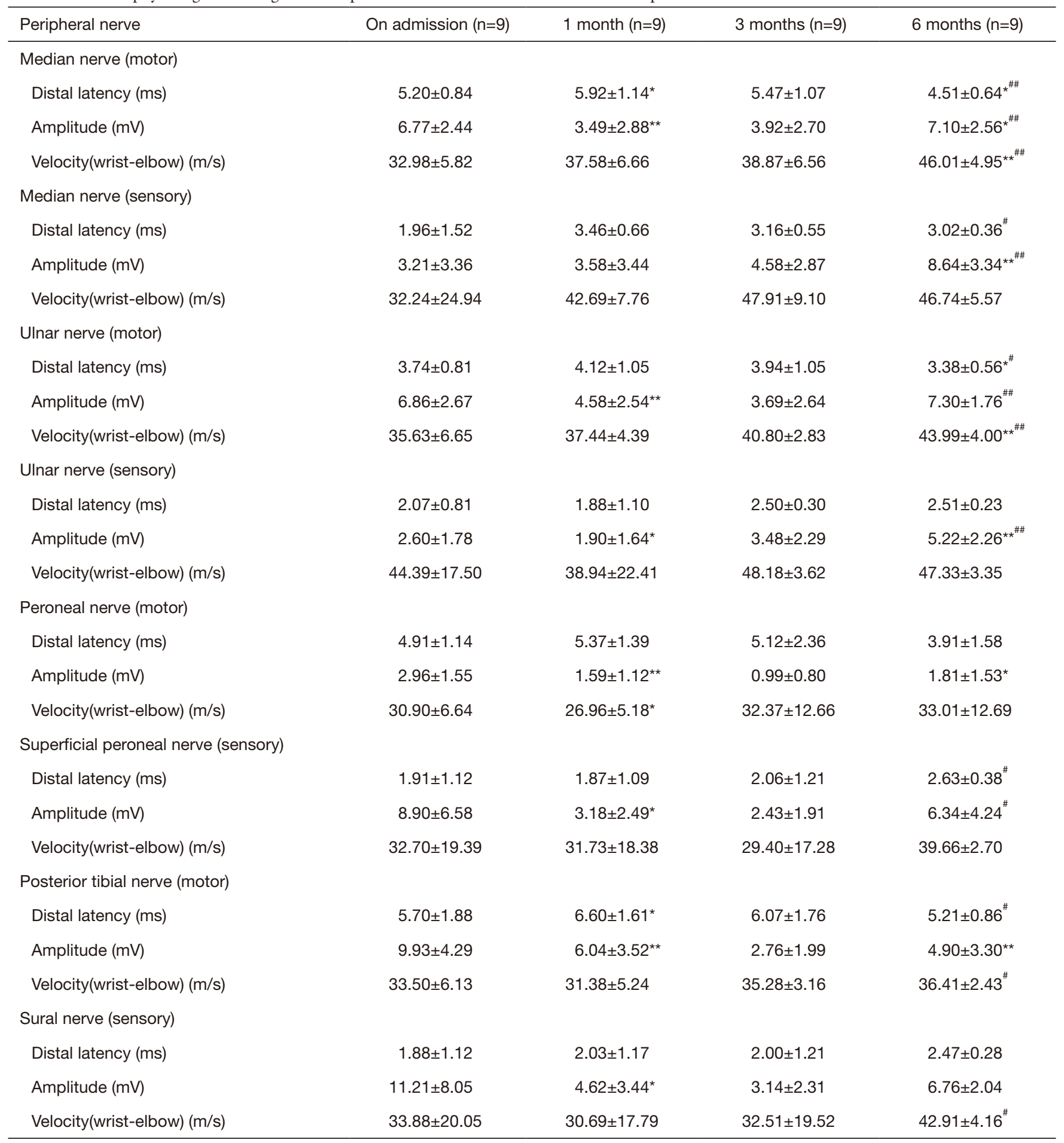

Data were shown as mean \pm standard deviation. $P$ values were calculated by Wilcoxon matched-pairs signed-rank test. ${ }^{*} P<0.05$, ${ }^{* *} P<0.01$ compared with "on admission" group; $\mathrm{P}<0.05$, "\# $\mathrm{P}<0.01$ compared with " 1 month" group. 
Also, patients went to the hospital several times with different treatments until they ended with n-hexane poisoning. Third, it takes a long time for individuals with chronic n-hexane toxicity to develop meaningful clinical manifestations. Nine patients were exposed to n-hexane for 2 to 8 months before the first signs of illness were clear. After the disease progressed for two months, some patients presented with numbness in both hands and legs, difficulty holding a bowl or wringing a towel, swelling of the legs, and difficulty climbing stairs. The polyneuropathy induced by $\mathrm{n}$-hexane poisoning was finally diagnosed with a compatible clinical history and normal clinical examination and routine electrophysiological examination findings. Fourth, because there are a lot of causes which can lead to the multiple peripheral nerve damage, such as the Guillain-Barre syndrome, amyotrophic lateral sclerosis, diabetes neuropathy, infectious multiple neuritis, and other toxicants (e.g., Furans, isoniazid, arsenic, trichloroethylene, chloropropene, acrylamide, organophosphorus). So it was difficult for doctors to make a diagnosis of $n$-hexane poisoning earlier, if the doctors were the first time to see a patient with polyneuropathy induced by $\mathrm{n}$-hexane, and the patients did not provide the exposure of n-hexane history at the same time.

It is essential to diagnose and administer treatment early, as the peripheral nerves have a limited capacity to regenerate, and the treatment may only affect the progression-not reverse the damage. In our study, none of the patients received an exact diagnosis at once, which is why some of their symptoms worsened. Both patients 6 and 7 were diagnosed and started physiotherapy on the same day, while the duration of disease for patient 6 was one month longer. Therefore, gait abnormalities were apparent in patient 6 , even though his sensory deficits were not as severe as those of patient 7 .

In our study, all patients worked in a poorly ventilated small workshop for several months and presented with neurological manifestations characterized by subacute, predominantly motor, polyneuropathy, and nerve conduction abnormalities that may meet the formal criteria demyelinating polyradiculoneuropathy. From the electrophysiological results upon admission and at the 1-, 3- and 6-month follow-ups, we determined that compared with the results upon admission, the results at the 1-month follow-up were significantly different in terms of the latency and amplitude of the median motor nerve signal, the amplitude of the ulnar motor and sensory nerve signal, amplitude and velocity of the peroneal nerve signal, the amplitude of the superficial peroneal nerve signal, DAL and amplitude of the tibial motor nerve signal, and amplitude of the sural sensory nerve signal $(\mathrm{P}<0.05)$. Therefore, we conclude that during the first month of clinical and physiotherapy, the nine patients' conditions worsened, mainly the severity of the motor nerve injury of the upper and lower limbs, according to the electrophysiological results. Patients with clearly established neuropathy had decreased motor and sensory conduction velocities, suggesting a demyelination process, although the primary pathology proposed was axonal swelling (2). The electrophysiological findings worsened at a slower rate after the patients were no longer exposed to n-hexane in our investigation, both in terms of sensory and motor function, consistent with the findings of several previous studies (2). Metabolism studies have suggested that n-hexane undergoes complex biotransformation, leading to 2,5-hexanedione (2,5-HD) formation, presumed to be the ultimate neurotoxic agent causes peripheral neuropathy. After n-hexane exposure is ceased, the accumulation of 2,5-hexanedione in the human body still causes significant pathological changes, including neurofilament recruitment, accumulation, winding, and gathering at the nodes Ranvier. Recent studies have shown that axon swelling or axon atrophy is the morphological hallmark of HD-induced neuropathy (3).

In this study, after six months of clinical and comprehensive physiotherapy, the electrophysiological results, including the DAL, amplitude, and velocity of the motor nerves, including the median, ulnar, peroneal, and tibial nerves, significantly differed $(\mathrm{P}<0.05)$ from the results upon admission or/and one month later. These results indicated that the motor nerves' recovery was superior to the sensory nerves; this finding is consistent with those in a previous study of 25 patients with chronic peripheral neuropathy induced by occupational n-hexane exposure (2). However, after six months, the motor conduction velocity of the median and ulnar nerves of 9 patients did not recover back to normal values, while 5 of 9 peroneal nerves and 4 of 9 tibial nerves recovered back to normal values, which meant the motor nerves of the lower limbs recovered more quickly than did those of the upper limbs. The SCV of 6 of 9 median sensory nerves, 8 of 9 ulnar nerves, 2 of 9 superficial peroneal nerves, and 7 of 9 sural nerves recovered back to normal values, which meant the sensory nerves recovered better than the motor nerves did. Another study of the electrophysiology of 25 patients found no significant difference in the sensory nerve conduction 
velocity after six months of treatment. After 12 months, the clinical manifestations had returned to normal values, but nerve conduction velocity had not yet returned to normal (2). Our results show that electrophysiology is a more sensitive indicator of peripheral nerve injury than clinical manifestations, even at the sub-clinical level.

The signs and symptoms caused by n-hexane were divided into three categories, mild, moderate, and severe, according to a guideline proposed by the National Occupational Health Standards of the People's Republic of China in 2017 (1). Also, we classified the patients from their ADL scores and electromyography presentations. Although 9 patients were divided into severe degree of n-hexane poisoning, but the rehabilitation evaluations, including the muscle strength of four extremities, the balance, the walking abilities and Barthel Index, which were more common used to reflect the function and self-care ability of patients, were different. In our cases, we integrated this information into an individualized rehabilitation program that included different treatment modalities, therapeutic exercise, nerve mobilization, gait training, occupational therapy, traditional Chinese medicine treatment, and patient education. The intensity of these interventions varied depending on the patient's functional status.

However, physiotherapy should be started as early as possible after patients are diagnosed to improve their functional status and life quality. In particular, after a patient becomes impaired, they may need to undergo physical therapy to help maintain muscle strength and avoid muscle cramping and spasms. The functional deficits of patient 1 , who had started a rehabilitation program five months after the onset of neuropathy, were more severe than were those of her colleagues, who started physical intervention immediately, even though she worked significantly fewer hours than did the other patients, 2 versus 8 hours, respectively. After peripheral nerve injury is diagnosed, a full subjective and aim examination must obtain a clear picture of how the lesion affects the patient. The examination should focus on pain, sensation and motor deficits, skin conditions, functional impairments, and activity limitations. And after 6 months rehabilitation, the recovery of walking ability, balance, and Barthel index of NO.1 patient, whose Barthel index score was 25 on admission, was poor than other patients. So it required a longer time of rehabilitation treatment for NO.1 patient.

Nerve injury can lead to pain, muscle weakness, or sensory deficits. Even though axons grow slowly, at a rate of $1 \mathrm{~mm} /$ day, reinnervation is possible. Rehabilitation for nerve injuries aims to protect the patients from added injuries and improve their function. Neuropathic pain is a typical result of nerve injury that can affect the quality of life. A multidisciplinary approach should be taken with drugs and different treatment modalities.

A consequence of denervation is muscle weakness and functional deficit. Therapeutic exercise has been proven to be much more effective in improving axon regeneration than have other physical therapy interventions (4). The efficacy of exercise regimens that enhance nerve regeneration depends on the upregulation of neuronal neurotrophic factors (5).

Therapeutic exercise and task-oriented practice must be combined meaningfully through available intervention programs. Therapeutic programs must be educative and motivational so that patients will exercise their full motor ability in their home and community activities. Understanding and applying these factors reflects a proper application of the sciences related to neurological rehabilitation and will supply patients with the most significant opportunity to attain functional competence. By understanding and applying these factors in our rehabilitation program, patients will have the highest potential for achieving functional competence.

The formulation of sports programs is especially important. For patients with peripheral nerve injury, exercise mode and intensity should be customized according to the patient's functional condition. In the initial stages, active resistance training is an effective way to increase the patient's muscle strength. According to our experience, active resistance training in patients with peripheral nerve injury needs to be appropriately increased in intensity and frequency to activate more neurons and muscle fibers, but importantly, it should be tolerated by patients. When a certain level of muscle strength is reached, it is necessary to focus on training patients' coordination and balance functions, and mainly low-intensity exercises with a high repetition rate improve patients' muscle endurance (6).

In our study, all patients reported experiencing motor and sensory dysfunction, which decreased their ability to work and quality of life. They needed to return to work as soon as possible, supplying early and correct diagnoses for treatments to be much more critical. Since n-hexane neuropathy is an occupational disease, employers must take proper measures to protect employees from exposure, for example, by implementing using gloves and masks.

$\mathrm{N}$-hexane neuropathy should be considered in diagnosing a patient with neuropathy, especially if the patient is 
chronically exposed to this chemical in a poorly ventilated workplace. Avoiding the toxin and exercises are efficient measures for patient rehabilitation. In many places where n-hexane is used, active measures are necessary to prevent severe toxicity. Exposure may occur by both inhalational and dermal routes, and thus, limiting inhalation and skin contact can prevent this condition.

\section{Conclusions}

We suggest that early general physical evaluation with electrophysiological examinations and comprehensive rehabilitation, including different modalities, therapeutic exercise, nerve mobilization, gait training, occupational therapy, traditional Chinese medicine treatment, and patient education, are essential so that patients can perform activities of daily living independently and return to work early.

\section{Acknowledgments}

Funding: This work was supported by the medical and health science and technology program of Zhejiang Province (2019RC172) to Yun Sun and the Natural Science Foundation of Zhejiang Province (LGF18H170002) to Zuobing Chen.

\section{Footnote}

Reporting Checklist: The authors have completed the STROBE reporting checklist. Available at http://dx.doi. org/10.21037/apm-20-2176

Data Sharing Statement: Available at http://dx.doi. org/10.21037/apm-20-2176

Conflicts of Interest: All authors have completed the ICMJE uniform disclosure form (available at http://dx.doi. org/10.21037/apm-20-2176). The authors have no conflicts of interest to declare.

Ethical Statement: The authors are accountable for all aspects of the work in ensuring that questions related to the accuracy or integrity of any part of the work are appropriately investigated and resolved. The study was conducted in accordance with the Declaration of
Helsinki (as revised in 2013). The study was approved by Clinical Research Ethics Committee of the First Affiliated Hospital, Zhejiang University School of Medicine (NO.: IIT20200475A) and informed consent was obtained from all the patients.

Open Access Statement: This is an Open Access article distributed in accordance with the Creative Commons Attribution-NonCommercial-NoDerivs 4.0 International License (CC BY-NC-ND 4.0), which permits the noncommercial replication and distribution of the article with the strict proviso that no changes or edits are made and the original work is properly cited (including links to both the formal publication through the relevant DOI and the license). See: https://creativecommons.org/licenses/by-nc-nd/4.0/.

\section{References}

1. Diagnosis of occupational chronic $\mathrm{n}$-hexane poisoning. GBZ 84-2017 ed: National Health Commission of the People's Republic of China; 2017.

2. Wang C, Chen S, Wang Z. Electrophysiological followup of patients with chronic peripheral neuropathy induced by occupational intoxication with n-hexane. Cell Biochem Biophys 2014;70:579-85.

3. Lopachin RM, Decaprio AP. Protein adduct formation as a molecular mechanism in neurotoxicity. Toxicol Sci 2005;86:214-25.

4. Gordon T, English AW. Strategies to promote peripheral nerve regeneration: electrical stimulation and/or exercise. Eur J Neurosci 2016;43:336-50.

5. Wilhelm H, Schabet M. The Diagnosis and Treatment of Optic Neuritis. Dtsch Arztebl Int 2015;112:616-25; quiz 626.

6. Streckmann F, Zopf EM, Lehmann HC, et al. Exercise intervention studies in patients with peripheral neuropathy: a systematic review. Sports Med 2014;44:1289-304.

(English Language Editor: J. Chapnick)

Cite this article as: Sun $\mathrm{Y}, \mathrm{Wu} \mathrm{X}$, Chen J, Wei S, Ji F, Wu R, Mao L, Bao W, Wen Y, Chen Z. The effect of rehabilitation in patients with polyneuropathy induced by occupational intoxication with n-hexane: a report of 9 cases. Ann Palliat Med 2020;9(6):4179-4186. doi: 10.21037/apm-20-2176 